\title{
L'Internet des diasporas noires aux États-Unis, en Grande-Bretagne et en France
}

The Internet of Black Diaspora in the United-States, Great-Britain and France

El Internet de las diásporas negras en Estados Unidos, Gran Bretaña y Francia

\section{Souley Hassane}

\section{(2) OpenEdition}

\section{Journals}

Édition électronique

URL : https://journals.openedition.org/remi/5045

DOI : $10.4000 /$ remi. 5045

ISSN : $1777-5418$

Éditeur

Université de Poitiers

Édition imprimée

Date de publication : 1 février 2010

Pagination : 119-139

ISBN : 978-2-911627-54-5

ISSN : 0765-0752

Référence électronique

Souley Hassane, "L'Internet des diasporas noires aux États-Unis, en Grande-Bretagne et en France », Revue européenne des migrations internationales [En ligne], vol. $26-n^{\circ} 1$ | 2010, mis en ligne le 01 février 2013, consulté le 15 avril 2022. URL : http://journals.openedition.org/remi/5045 ; DOI : https://doi.org/ 10.4000/remi.5045 


\title{
Note de recherche
}

\section{L'Internet des diasporas noires aux États-Unis, en Grande-Bretagne et en France}

\author{
Souley HASSANE*
}

\section{INTRODUCTION}

La multiplication des sites communautaires, des forums, des sites de rencontres et de blogs transforme la production de l'information dans les diasporas noires. L'internaute de Harlem, Chicago, Atlanta, peut se « rendre » à Niamey, Lagos Harare, Kingston et Brixton en naviguant sur la toile. Il se crée des routes, des circuits, des passages obligés, des outils de transports et des manières de circuler dans l'espace Internet. Dans cet « espace plat » (Friedman, 2001), les acteurs circulent sans contrôle et sans papiers : la navigabilité est fluide et elle bouscule et déplace les frontières physiques. Mais l'Internet a aussi ses codes, ses règles et ses modes de régulation que les usagers contribuent aussi à construire.

L'historique des navigations et la traçabilité des usages de l'Internet par les membres des diasporas montrent un «cyberespace communautaire » (Alkalimat, 2006), un « espace virtuel diasporique » (Karim, 2001), une « communication diasporique » (Verhust,1999), et des « diaspora online» (Bernal, 2006) qui n'ont de sens que par une occupation spécifique du cyberespace. Comment rendre compte de cette occupation de l'espace virtuel ? Quels sont les repères topologiques des espaces sans « lieu »? Comment caractériser et catégoriser les productions identitaires dans des contextes économiques et politiques différents ? Il s'agit dans cet article de cartographier ce que nous appelons « les thématiques identitaires » de l'espace virtuel occupé par les diasporas noires à partir de leurs sites Internet. Cartographier le virtuel veut dire, ici le rendre visible, en répertoriant les lieux à partir desquels les individus s'expriment. Cette cartographie repose sur une représentation graphique de ces sites Internet mais aussi des contenus susceptibles de « concrétiser » leur caractère virtuel. Les graphiques sont réalisés grâce à deux logiciels Gephi ${ }^{1}$ et TouchGraph ${ }^{2}$. Le premier

* Université de Poitiers, Minoritymedia, Migrinter, souley.hassane@univ-poitiers.fr

1 Ghephi a été développé par Mathieu Bastian du Laboratoire TIC Migrations, Paris.

2 TouchGraph a été développé par Alex Shapiro à partir de la version initiale du Google Graph Browser de Christian Langreiter, basé sur l'applet Java Vanilla-Vista. Cf. www.touchgraph.com. 
permet une représentation graphique à partir d'un corpus de sites générés autour d'un site donné et grâce au moteur de recherche NaviCrawler ${ }^{3}$. Dans ce travail, nous utilisons TouchGraph parce qu'il permet de passer outre la constitution d'un corpus. Il permet une représentation directe de l'activité autour d'un site Internet. Il permet également de dé-sélectionner tous les sites sans rapport avec une thématique. Enfin, il permet de limiter la taille du graphe et rendre ainsi l'activité Internet lisible et représentable. Il s'agit moins de placer des points dans un repère classique que de faire une typologie et une topologie d'une masse d'informations. Les graphes ne sont pas des cartes même s'ils nous aident à représenter le virtuel. L'objectif que nous poursuivons est donc de représenter des espaces d'expressions identitaires sur la base d'un relevé topographique des sites Internet, de leurs contenus et des acteurs qui les animent. L'Internet a alors une double fonction, celle d'objet d'étude et de terrain d'enquêtes, d'où la mobilisation de données empiriques sur les diasporas noires pour mieux comprendre leur occupation du cyberespace. Dans cet article, nous nous appuyons principalement sur les sites américains, français et britanniques.

Les populations que nous étudions sont des « diasporas de victimes » et des « diasporas culturelles » (Cohen, 2006 : 42). Selon Hall (1993) la traite transatlantique marque leur déracinement historique et traverse les expériences culturelles identitaires qui prolongent leur histoire dans le monde occidental. La dispersion de l'Afrique vers l'Europe et les Amériques, une mémoire collective sur la terre d'origine, l'idéalisation de la terre ancestrale, l'idéologie du retour, une forte conscience d'appartenance ethnique, une relation complexe avec les sociétés d'accueil, une empathie et une solidarité entre les membres et la possibilité d'une vie créative et distinctive riche dans les sociétés d'accueil sont les éléments qui les caractérisent comme diasporas (Cohen, 2006 : 43). La revendication de l'identité africaine se traduit par des modes contradictoires d'affiliation, de reconnections et de reconstruction ; la création de médias communautaires, l'histoire, la religion, la solidarité et la politique sont autant de modalités de « retour à l'Afrique ». La notion de « diasporas noires », dans ce travail, a aussi le sens métaphorique d' "atlantique noir»(Gilroy, 2003) qui appréhende la complexité des processus transnationaux, globaux ou glocaux des expériences de réinventions identitaires et culturelles (Bhabha, 2007).

\section{TOPOGRAPHIE DES SITES INTERNET}

En Europe et aux États-Unis, l'activité médiatique des diasporas noires est très importante : on recense entre 2006 et 2009, au moins 500 médias ${ }^{4}$ créés en Europe dont 185 presse Internet, 163 sites presse papier, 140 radios, 41 télévisions, 4 radios-internet et 4 télévisions-internet. $60 \%$ de ces médias sont commerciaux. Plus de $80 \%$ de tous

3 Navicawler a été développé par Mathieu Jacomy du Laboratoire TIC Migrations, Paris.

4 Enquêtes réalisées par Minoritymedia une équipe européenne d'excellence ( $\left.6^{\circ} \mathrm{PCRDT}\right)$ sur les médias des minorités ethniques. Elle est hébergée par le laboratoire MIGRINTER (CNRS) à l'Université de Poitiers. Composée de sept chercheurs, l'équipe a procédé au recensement des médias des minorités dans huit pays européens (France, Grande-Bretagne, Italie, Espagne, PaysBas, Hongrie, Turquie et Allemagne) ainsi qu'une analyse de leur contenu (www.minoritymedia.eu). 
ces médias utilisent la langue majoritaire dans la production de l'information. $78 \%$ acceptent la publicité et les financements des annonceurs qui ciblent les communautés noires. Aux États-Unis, nous travaillons sur trois bases de données africainesaméricaines qui enregistrent plus de 2000 sites regroupés sur http://www.msoyonline.com, http://www.blackwebsites.com et http://www.blackpressusa.com. Les sites Internet créés par les diasporas noires en Europe ne représentent, quantitativement et qualitativement, qu'une infime partie des sites africains-américains. Si on y ajoute les 32 millions de blogs africains-américains recensés par « google blog search», les États-Unis pèsent d'un poids écrasant sur les cyberinitiatives des diasporas noires. L'Amérique domine aussi par la richesse des sites, des sujets traités et des contenus. Globalement, les sites Internet américains s'imposent comme modèles et surtout comme tête de pont dans la production et la diffusion des «thématiques identitaires ». Essentiellement commerciaux, ils utilisent exclusivement l'Anglais. Dans leur majorité, ces sites s'adressent aux communautés noires locales et nationales. Ils traitent de sujets de proximité à l'exception de thèmes comme l'esclavage, la colonisation, les conflits en Afrique, etc.

L'Internet des diasporas noires est tributaire de son contexte socio-économique. Son idéologie s'articule autour des identités et appartenances noires et de la réappropriation des paradigmes du néolibéralisme. Les entreprises commerciales, les bureaux d'affaires contrôlent ce cyberspace et lui donnent ainsi son caractère commercial, marchand et néo-libéral. Les promoteurs qui ont su l'utiliser pour se faire une place dans l'économie nationale disposent d'un capital financier et d'un capital culturel qui participent à une marchandisation des identités. Ainsi dans une logique goffmanienne, les «porte-identités» (Goffman,1974: 76) en quête de normalité bricolent les « identités statutaires », (De Singly, 2005 : 123), les «identités biographiques », « immédiates » et narratives » (Kaufman, 2001 : 167). En cela, ils font preuve d'une réelle créativité. Ils bricolent les identités en fusionnant marché, appartenances et informations. Ils agissent à partir de leurs vécus et de multiples histoires (esclavage, colonisation, racisme, migrations etc.) pour formuler une perspective nouvelle.

Des usages de l'Internet des communautés noires étasuniennes, françaises et britanniques on peut dégager plusieurs niveaux qui correspondent à des moments et des modes de production et d'appropriation de l'Internet. Le premier niveau est celui d'un "Internet $d u$ je ». Celui-ci est caractérisé par toutes les utilisations personnelles et personnalisées des nouvelles technologies et de l'Internet. Les sites de rencontres communautaires, les boutiques en ligne et les réseaux sociaux communautaires constituent le lieu de cet «Internet du je ». L'internaute se présente comme le sujet qui choisit ses interlocuteurs, ses partenaires et ses marchandises. Il dépose ses données personnelles, ses informations bancaires, son profil d'internaute communautaire, de client, de partenaire et ami potentiel. L' «Internet du je » est aussi un jeu ou le sujet se cache derrière des pseudonymes et se découvre à travers ses goûts, ses choix et ses fréquentations. Les sites de rencontres en sont l'exemple tels blackintroductions.com, Blacksingle.com, blacklove.com.

L' «Internet $d u$ “nous" directif » est représenté par l'ensemble des sites Internet communautaires dominants et légitimes. Ces derniers ont été créés au début de l'Internet et constituent aujourd'hui les précurseurs de l'espace virtuel noir. Ces sites 
commerciaux ou associatifs ont été pourtant identifiés aux populations noires. Leur contenu traitait de discrimination, d'égalité, de justice sociale, d'histoire, de culture et de célébration. Le traitement de ces questions a transformé les sites en pourvoyeurs d'informations générales pour des communautés. En effet, dans les trois pays concernés par notre recherche, les internautes se réfèrent à ces sites qui écrivent pour la « communauté noire ». C'est un «nous » directif en ce sens qu'il ne se discute pas par les internautes. Il n'y a pas débats entre les lecteurs et les concepteurs des contenus. Ces sites traitent des communautés sans leur mandat avec le présupposé que c'est dans l'intérêt de celles-ci. En outre ils comblent le manque d'une information produite par les diasporas noires et pour elles-mêmes. Le site www.blackinamerica.com en est l'illustration. Il est directif dans le sens où il indique la marche à suivre à la communauté et son caractère pédagogique lui donne en quelque sorte une dimension messianique. C'est aussi le cas des sites comme www.100blackmen.org, www.aapci.org, www.besomeone.org, www.blackculinarians.com, www.bignet.org, www.blackwallstreet.org, www.bisa-hq.org, www.jack-and-jill.org, www.missblackusa.org, www.nacme.org, www.nationalactionanetwork.net, www.naacp.org, www.nabhood.org, www.nabainc.org, www.nabcj.org, www.namdntl.org, www.nbcdi.org,www.nsbe.org, www.nul.org et www.rainbowpush.org.

L'existence de ces sites est souvent perçue comme la réparation d'une injustice sociale en promouvant le droit à l'information pour les minorités. En l'absence d'autres acteurs, ces sites deviennent une autorité morale et prétendent même incarner la communauté face à la société dominante : " premier site noir d'information », « premier site noir sur les affaires », « premier site noir sur les femmes » etc.

L' « internet du nous participatif » représente l'ensemble des forums communautaires. Première forme d'interactivité collective, ils permettent aux internautes de discuter et de donner leur avis sur différents sujets. Le " forum social» Blackplanet.com (États-Unis) place l'internaute au centre de l'interaction. Mais ces sites se présentent aussi comme une ressource symbolique et marchande : la publicité, la vente d'espace personnel, la suggestion des liens commerciaux, etc.

Ainsi, l'Internet participatif donne à l'internaute les ressources de sa « transformation » en un personnage autre. L'anonymat des pseudonymes, la gratuité et la facilité des inscriptions, la possibilité de se construire une "identité », les outils d'investissement de l'espace public virtuel (photos, vidéo, mise en page minimale, chat sur téléphone portable, sélection de groupes d'amis, sélection de gens par centre d'intérêt, sélection des interlocuteurs par profession etc. ) et l'absence d'engagement sont autant de ressources qui motivent les individus et fait vivre ces sites. Des personnes « muettes » dans la vie réelle, deviennent loquaces et actives derrière leurs claviers.

$L^{\prime}$ "Internet de soi » représente des milliers de blogs créés sur diverses platesformes qui affichent une «identité noire » sur la place publique. Les bloggeurs noirs sont estimés à plus de 10 millions aux États-Unis. Ils constituent, aujourd'hui, une ressource importante dans la fabrication des «buzz » et des «e-influence », des « $e$ autorités » et de la «link-influence ». Ils influencent les opinions sur le net en raison de 
leur nombre et de leur activité. La blog-influence devient ainsi une ressource entre les mains de «petits porteurs de l'information ». Parmi les «black bloggeurs », on peut distinguer les «bloggeurs influents » et les « réceptacles d'influence ». Les premiers sont reconnus comme des sources d'informations sérieuses et pertinentes tandis que les seconds sont des «amplificateurs puissants ». Les blogs des communautés noires donnent l'information du point de vue des individus et la rendent publique. Ils sont en quelque sorte la face «égocentrique » de l'information communautaire. On a là un vaste mouvement qui peut devenir un contre-pouvoir médiatique important et influent dès lors qu'il peut s'opposer aux médias dominants et aux médias communautaires dominants. L'identité noire et ses formes d'appartenances sont au cœur de ces blogs.

On notera que tous ces sites constituent un corpus important des médias électroniques. Pour des communautés noires marginalisées, cette "cyber-créativité » constitue un moment et un lieu important de l'histoire des populations noires d'Occident. On est d'ailleurs frappé par le fait que les sites sont envisagés comme une réponse à un problème et une justification. Bien plus, les articles, éditoriaux et postings ont un caractère fortement démonstratif. Cette tension argumentative permanente traverse toutes les formes de sites et alimente toutes les interventions dans l'espace virtuel. La liberté d'information est invoquée comme le principe fondateur de ces médias. C'est du reste la matrice de toutes les thématiques identitaires. L'héritage de l'esclavage, les discriminations et la domination économique, sont des thèmes qui relèvent de toutes les pratiques médiatiques. Ces sujets occupent de manière redondante l'espace virtuel au point de représenter pour de nombreux internautes un mode d'entrée en scène. Abdul Alkalimat, initiateur des e-black studies à l'Université de Toledo (États-Unis) écrit que l'Internet de ses communautés instaure « une cyberdémocratie, une intelligence collective et garantit la liberté d'expression. » (2006: 105). L'appropriation de l'Internet montre selon lui «comment une communauté exclue socialement peut se transformer en communauté connectée, capable de mobiliser un cyberpouvoir pour faire avancer ses intérêts. La transformation d'une communauté historique en une communauté connectée est catalysée par les cyberorganisateurs et par l'organisation et la mobilisation du contenu et de l'interactivité du cyberespace. » (Alkalimat, 2006 : 107).

Autrement dit, des groupes encore relégués à la rubrique des faits-divers, des scandales et des affaires judiciaires s'emparent de mots et de phrases, reviennent à l'écriture, la lecture et la documentation, pour se rendre visibles sur la place publique. $\mathrm{Au}$ passé synonyme de silence et d'invisibilité succède un présent où des voix, discordantes de celles des dominants et discordantes entre elles, se font entendre. Dès lors on peut se risquer à dire que l'importance quantitative et qualitative n'est pas qu'un « effet » de la structure de l'Internet mais que les conditions socio-économiques et la domination subies par les différents cyberacteurs permettent d'expliquer significativement le phénomène. La compréhension de cette dynamique implique, aussi la prise en compte des conditions réelles des usagers. L'Internet des populations noires de l'hémisphère nord n'est pas simplement un acte techniciste ou médiatique, c'est aussi un usage contre-hégémonique dans bien de ses aspects. On ne peut faire l'économie de cette dimension si on veut comprendre ce qui s'expose sur le net. La lutte contre les discriminations et les stéréotypes, la lutte contre toutes les formes d'exploitations est le ressort des discours produits. 


\section{TYPOLOGIE DES SITES INTERNET}

La typologie des sites Internet noirs en France, aux États-Unis et en GrandeBretagne correspond à une certaine réalité socio-économique. Ils reflètent une activité associative, communautaire ou publique. La typologie des sites renvoie dans une certaine mesure à une topographie identitaire. Les questions identitaires prennent, dans ces conditions, une dimension sociale et politique. On peut les regrouper en dix groupes distincts et pertinents.

Le premier groupe est celui des sites Internet de la presse écrite et audiovisuelle destinée aux populations noires de ces pays. En effet, les médias créés par ces minorités disposent de sites-vitrines qui les présentent. Ils sont complémentaires au média écrit ou analogique et apportent une certaine densité à l'espace communautaire virtuel. L'Internet est de plus en plus le lieu du stockage des archives et des podcasts, autre moyen de fidéliser les clients et de toucher un public nouveau. Ces médias tentent ainsi de combler le manque à gagner créé par l'avènement de l'Internet et d'une nouvelle économie de l'information. Le développement de ces sites est inégal dans la mesure où certains sont des « machines » commerciales tandis que d'autres sont encore, techniquement, à un stade associatif. L'espace des médias d'informations générales destinées aux Noirs reste néanmoins très fréquenté. C'est un espace de la publicité et de services dont la plus-value financière renforce le media principal. Blackpressusa.com est une plate-forme qui regroupe les sites de la presse écrite et de tous les métiers de la presse notamment les instituts, les centres de formation et les maisons d'éditions (Graphe 1). Le site est connecté à toutes les grandes associations noires. Cette organisation est reprise par le syndicat des journalistes noirs de GrandeBretagne à travers son site www.nuj.org. La situation est différente en France malgré

Graphe 1 : Les sites Internet de la presse écrite et des métiers de journalisme

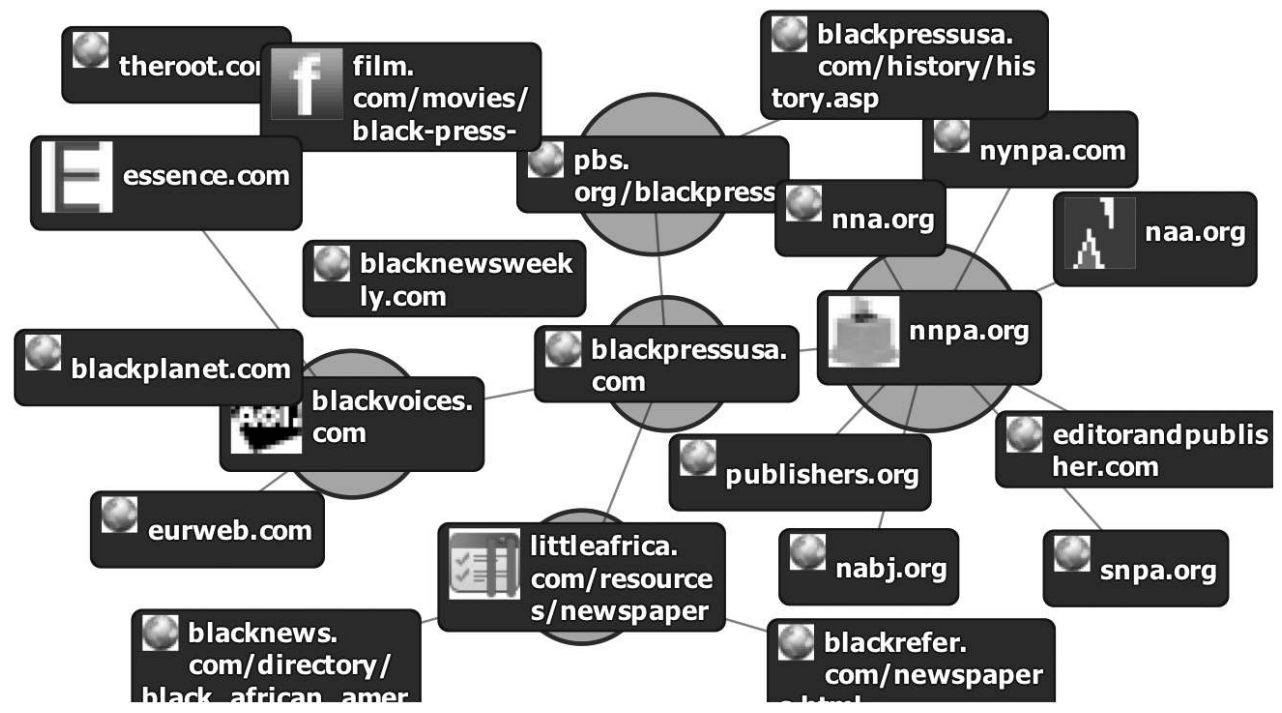


une forte production médiatique, les diasporas ne disposent pas de telles plates-formes. Chaque média développe son propre cyberespace connecté, le plus souvent aux sites des diasporas noires américaines.

Le deuxième est l'espace virtuel occupé par les sites des organisations et associations communautaires. Ces sites représentent les principales organisations noires. L'exemple des grandes organisations est suivi par les associations locales qui font de l'existence sur le web un outil de visibilité et d'ouverture sur le monde. L'enjeu de visibilité et de réaffirmation identitaire est fort dans tous les pays. Une fois encore, le site Internet, porteur des questions identitaires, est le reflet d'une réalité bien concrète. Les questions identitaires sont au cœur de la présence des diasporas noires sur Internet. Le site de ligali se définit ainsi : " Identifier et revendiquer notre identité originelle, notre culture et notre histoire est une part importante du développement émotionnel, spirituel, économique et mental de la communauté africaine dans la société occidentale et sur le continent africain. Nous pensons fortement, que chaque individu a le droit de s'autodéfinir en fonction de ses principes, de ses valeurs sociales et de sa culture. C'est pour cette raison que nous affirmons et embrassons notre identité africaine et notre héritage comme quelque chose de positif et essentiel pour notre progrès individuel et collectif mais aussi pour notre progrès politique. Nous rejetons, par conséquent, le terme «noir » comme un concept marqué qui vient d'une culture qui a historiquement exploité le peuple africain et l'oppresse et exploite aussi son africanité. Le terme « noir » déconnecte les Africains d'Afrique et les force à devenir homogène et une masse indescriptible. ${ }^{5}$ Ces sites portent la militance panafricaniste et afrocentrique sur le cyberespace ${ }^{6}$.

Le troisième groupe aux États-Unis est celui des sites qui traitent de l'éducation. On y distingue les sites des écoles noires (ce n'est pas encore le cas en GrandeBretagne et en France). Mais aussi les sites des institutions qui accordent des bourses et des soutiens de toutes sortes aux institutions scolaires. Le premier est un annuaire des écoles communautaires noires aux États-Unis. En Grande-Bretagne et aux États-Unis, elles enregistrent un taux de réussite scolaire et académique faible par rapport aux autres minorités et un taux d'exclusion scolaire important. En Grande-Bretagne, la question de l'exclusion des « garçons noirs » a fait l'objet de nombreux rapports et études (Rex, 1986, 1998 ; Modood, 2009) montrant que l'école est une préoccupation centrale dans ces communautés. Dès lors les questions d'éducation et de scolarité occupent une place importante sur le net et sont devenues une thématique majeure pour les différentes communautés ${ }^{7}$.

5 www.ligali.org

6 www.100blackmen.org, www.aapci.org, www.aasb.net, www.besomeone.org, www.blackculinarians.com, www.bignet.org, www.blackwallstreet.org, www.bisa-hq.org, www.jack-and-jill.org, www.missblackusa.org, www.nacme.org, www.nationalactionanetwork.net, www.naacp.org, www.nabhood.com, www.nabainc.org, www.nabcj.org, www.namdntl.org, www.nbcdi.org, www.nsbe.org, www.nul.org, www.rainbowpush.org

7 Les pricipaux sites sur le thème de l'éducation : www.morrisbrown.edu, www.morris.edu, www.nsu.edu, www.ncat.edu, www.nccu.edu, www.oakwood.edu, www.paine.edu, www.pqc.edu, www.philander.edu, www.pvamu.edu, www.rustcollege.edu, www.st-aug.edu, www.saintpauls.edu, www.savstate.edu, www.shawuniversity.edu, www.sheltonstate.edu, 
Le quatrième groupe regroupe tous les sites qui traitent des questions familiales. Les sites sur les «familles noires », les « mères noires », les « enfants noirs », les « filles noires » etc. sont importants. Ils font référence, régulièrement à des centres et structures de consolidation de la «famille noire ». Un de leurs enjeux est d'accompagner les jeunes familles, les jeunes mères et pères afin de renforcer la responsabilité parentale. Si la reproduction des familles est un enjeu central pour les minorités, la décroissance de la population noire américaine fait aussi l'objet de débats dans les communautés locales et nationales. L'épanouissement des «black kids » (enfants noirs) et des «black youth » (jeunes noirs) est au cœur des préoccupations de ces communautés dont les conditions économiques sont modestes et parfois misérables. Le «women empowerment» accompagne toutes ces initiatives autour de la famille alors que les femmes, considérées comme pilier de la famille sont au cœur du dispositif communautaire et national de lutte contre toutes les formes de précarités et d'abandon social. Les sites de célébrations des fêtes familiales comme le Kwanzaa s'inscrit dans le projet idéologique et identitaire de maintenir la famille, mais aussi, de procurer à ses enfants et à ses membres des célébrations authentiques et différentes. Célébrée depuis 1966, la fête de Kwanzaa est l'équivalent de noël dans les diasporas noires d'Amérique et d'Europe. Son fondateur Maulana Karenga écrit sur le site officiel : "L'intérêt de ce site est de fournir une information qui révèle et démontre l'intégrité, la beauté et le sens des célébrations et de ce fait aide à approcher le Kwanzaa avec une profondeur de la pensée, une dignité et un sens du fait spécial qu'il mérite $»^{8}$. Cette authenticité reinventée et recréée traverse toute l'histoire des Noirs dans le monde occidental et aux États-Unis tout particulièrement.

La question de la transmission et des liens intergénérationnels fait aussi partie des projets de ces communautés et sont l'objet de nombreux sites, de même que ceux consacrés aux «elders », les anciens des communautés, les retraités et les personnes âgées. En France les sites américains sont des ressources et sur plusieurs sites généralistes des communautés noires de France ${ }^{9}$ on retrouve les mêmes sujets, ceux liés à la famille, aux enfants et aux femmes.

La cinquième catégorie de sites Internet traite de la santé, de la couverture médicale et en particulier des structures médicales : hôpitaux, cliniques et centres de soins communautaires. Cet espace sanitaire virtuel reflète la précarité des communautés noires mais ils cherchent aussi à dénoncer la stigmatisation des "maladies ethniques ». D’autres sites sont consacrés aux médecines dites alternatives : les régimes alimentaires « africains », le « yoga africain » et la « nourriture minérale »

www.shorter.edu, www.scsu.edu, www.sus.edu, www.subr.edu, www.susla.edu, www.swcc.edu, www.spelman.edu, www.stillman.edu, www.talladega.edu, www.tnstate.edu, www.texascollege.edu, www.tsu.edu, www.tougaloo.edu, www.trenholmtech.cc.al.us, www.tuskegee.edu, www.uapb.edu, www.umes.edu, www.utep.edu, www.udc.edu, www.uvi.edu, www.vsu.edu, www.voorhees.edu, www.wvstateu.edu, www.wilberforce.edu, www.wileyc.edu, www.wssu.edu, www.xula.edu

8 http://www.officialkwanzaawebsite.org/index.shtml

9 www.grioo.com, www.afrik.com, www.afrikra.com, www.africamaat.com, etc. 
sont souvent valorisés et font l'objet d'une réappropriation identitaire : Le site http://queenafua.moonfruit.com/ de Queen Afua et http://www.drsebi.com/ du Docteur Sebe sont une référence en Grande-Bretagne et aux États-Unis.

La sixième catégorie regroupe tous les sites qui traitent de l'histoire, de la mémoire et de l'héritage africain-américain. Ces thématiques recouvrent toutes les phases de l'histoire noire américaine, allant de la période de l'esclavage à la situation contemporaine. Dans ces sites, l'histoire des Africains-Américains commence en Afrique. Ce positionnement est la fois historique et idéologique. En établissant une continuité entre les deux continents, il s'agit de répondre à la société dominante pour dire que l'histoire des anciens esclaves est celle d'hommes arrachés à leur continent et à leur terre et de demander que le «voyage » (The journey), que les acteurs noirs traduisent par arrachements, exils et déportations, soit reconnu. L'enjeu de ces sites est de lutter contre une forme d'amnésie historique et idéologique qui tendrait à couper les afrodescendants de l'Afrique pour en faire des individus sans racines (ou à racines multiples). Si ces thèmes ont fait et font encore l'objet de débats importants et récurrents dans les milieux universitaires, ils sont aujourd'hui entendus notamment dans le cadre des Black studies. Reste que la vie sous l'esclavage dans l'Amérique rurale post-esclavagiste et la vie urbaine des Africains-Américains sont encore largement traitées par ces sites d'histoire ou ceux dédiés aux musées, aux associations, aux intellectuels, aux universitaires, aux institutions de recherches, etc. L'histoire et la mémoire ont aussi fait irruption en France et en Grande-Bretagne dans l'espace virtuel. Les Britanniques sont les premiers à s'approprier les thématiques historiques américaines en raison de la langue et des liens anciens entre intellectuels et activistes des deux pays. Le Black History Month (BHM) est célébré aujourd'hui dans les deux pays. Selon ses créateurs :

«Le mois de l' histoire des Noirs se tient annuellement en octobre en GrandeBretagne et en février aux États-Unis et au Canada. L'objectif de ce mois est de : 1. Promouvoir la connaissance de l' histoire des Noirs et de leur héritage culturel.

2. Élever la confiance et la conscience des populations noires sur leur propre héritage. 3. Diffuser la contribution positive des Noirs dans la construction de la société britannique ». ${ }^{10}$

Le Black History Month est un mois de conférences, de débats, de discussions, de leçons d'histoires, de partage d'expériences et de témoignages sur l'expérience des Noirs en occident et nombreux sont les sites consacrés à ces manifestations. Il n'existe pas en France de sites de cette ampleur et si l'idée d'un mois de l'histoire des Noirs a été discutée en 2005 elle n'est plus à l'ordre du jour. On ne trouve qu'un seul site d'histoire : africamaat.com, qui tente de s'inspirer des initiatives britannique et américaine. De façon générale les auteurs de ces sites présentent leur histoire comme double, hybride, métissée, mélangée, bigarrée, multiple ou diverse. Il s'agit de mettre l'Afrique au cœur des identifications au même titre que d'autres appartenances. Ces sites illustrent parfaitement le débat récurrent qui traverse ces sociétés. La question du

10 www.blackhistorymonth.org.uk 
« choc des civilisations » aux États-Unis et celle de l'identité nationale ont convoqué l'histoire sur la place publique pour réinterroger non seulement les appartenances mais surtout la loyauté des uns et des autres. La «droitisation » des États-Unis sous l'influence des néo-conservateurs et la montée d'un « capitalisme du désastre » (Klein, 2008), perçues comme un contrôle des ressources, une domination politique et financière des pays de l'hémisphère Sud, ont participé au "retour de l'histoire » sur la place publique. La résistance des «communautés » se fait souvent par un retour sur leur identité et la réaffirmation violente ou pacifique de leurs expériences. Les sites britanniques par exemple en sont une illustration. (Graphe 2).

Graphe 2 : Sites Internet sur la célébration de l'histoire des diasporas noires britanniques

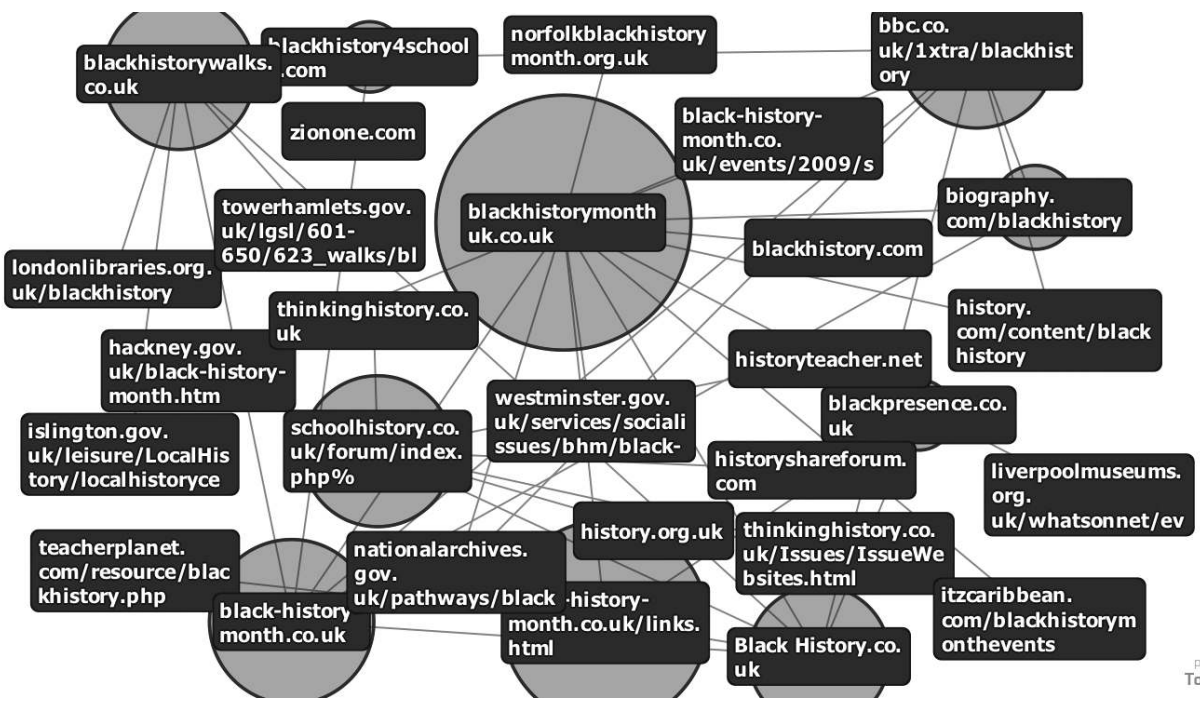

TouchGraph

Le septième groupe est composé de sites religieux. L'Internet est pour les «black churches » considéré comme un «véritable don de Dieu » ${ }^{11}$. L'identité noire chrétienne est interprétée par les nouveaux évangélistes, les pentecôtistes de la nouvelle église. Inspirés par les télévangélistes américains, ces acteurs chrétiens utilisent toutes les technologies pour diffuser le nouveau christianisme fondé sur la « re-naissance », l'idéologie du « born-again » (Mary et Cortens, 2000).

Les «black churches » de Grande-Bretagne sont exemplaires à plus d'un titre (Graphe 3). Les sites Internet développent une panoplie de services à la personne :

11 www.eauk.org, www.kicc.org.uk, www.cftchurches.org, www.cgwf.org, www.hotric.org.uk, www.mountainoffire.org, www.ntcg.org, www.peoministries.org, www.jesushouse.org, www.ruach.ministries.co.uk, www.trinytubaptist.org.uk, www.graceoutreach.com, www.aceauk.org, www.christembassy.org, www.faithinbusiness., etc. 
Graphe 3 : Les églises noires britanniques sur internet

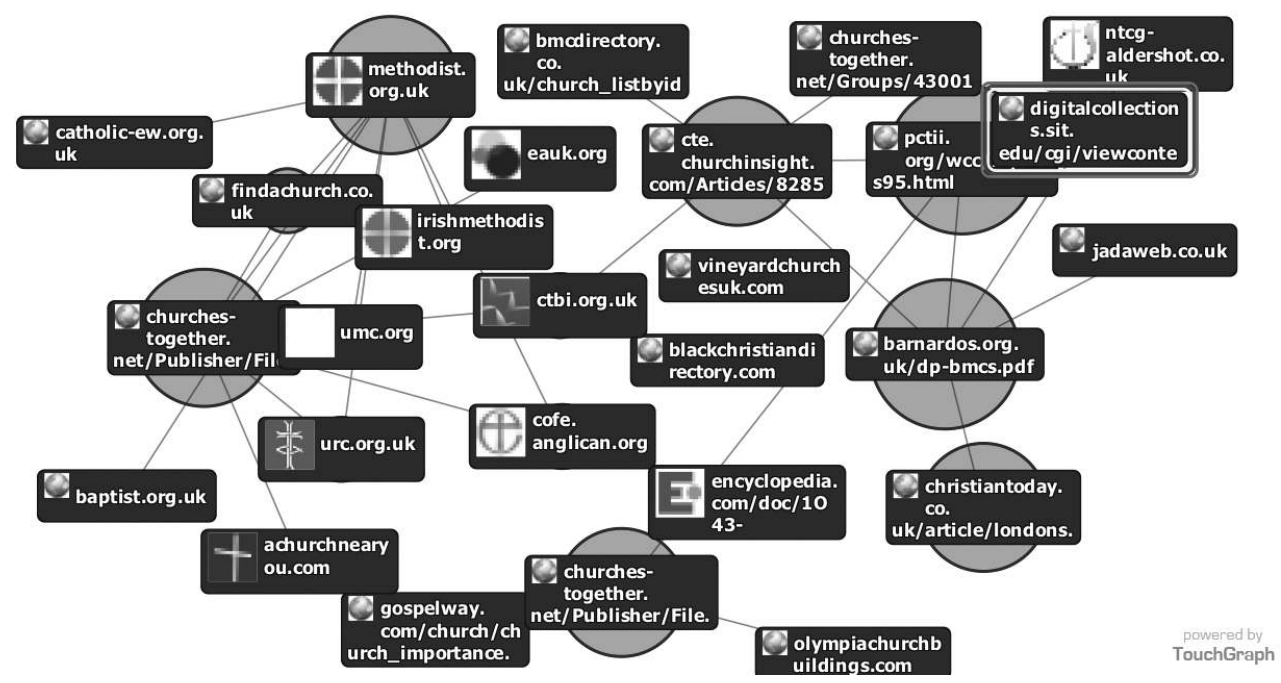

e-conversion, e-prédication, e-learning chrétien, e-confession, e-prières, ebénédictions à destination du cybernaute. Les cyberpreachers tentent de répondre aux questions éthiques, morales, familiales, funéraires et sanitaires. Tous les sites réaffirment leur vision de re-convertir leurs visiteurs comme l'écrit le Pasteur Kingsley Appiagyei de Trinitytubaptist org.uk : "Développer une assemblée de véritables croyants engagés à démontrer un véritable amour chrétien aux gens heurtés, déprimés et frustrés afin de les renforcer pour influencer leurs familles et le monde dans le culte et la vérité ».

Les « sites de Dieu » (Godnet) sont des vitrines de la communication et aussi des églises virtuelles. L'idéologie qui accompagne ces églises est inscrite dans la théologie de la prospérité (Fath, 2008). L'église interprète la richesse comme un signe de la grâce divine : gagner de l'argent est un acte divin (Fourchard, Mary et Otayek, 2005). En effet, la théologie de la prospérité devient un idiome du néolibéralisme mis en œuvre par les néo-conservateurs américains.

Les discours qui légitiment la production de l'information sur les sites musulmans font appel à la notion de «communauté musulmane ». "L'islam de marché » (Haenni, 2005) y est largement dominant. Les médias de Nation of Islam constitue une plate-forme importante et vise comme tous les sites religieux à convaincre et faire adhérer le lecteur. Le media Final Call s'inscrit dans cette rhétorique : "La version Internet de Final Call (Dernier Appel) avait commencé comme un simple outil de promotion développé par les élèves de la Nation de l'islam à l'occasion de la Marche d'un Million d'Hommes en 1995. Recevant des millions de visites depuis sa mise en ligne, il a évolué comme le compagnon électronique de la version papier de Final Call dans la tradition des publications de la nation de l'Islam. La version électronique de 
Final Call a pour objectif d'être la source d'information de ceux qui ont soif des reportages sans compromis dans le paysage actuel des médias orientés. " 12 Malgré un discours radical, Finalcall.com est lié aux principaux sites de la communauté noire américaine (Bet.com, Blackamerica.com, Blackcommentator.com, Blacknews.com, naacp.com ) mais aussi à des sites chrétiens (Voir le Graphe 4).

Graphe 4 : Les Black Muslims américains sur Internet

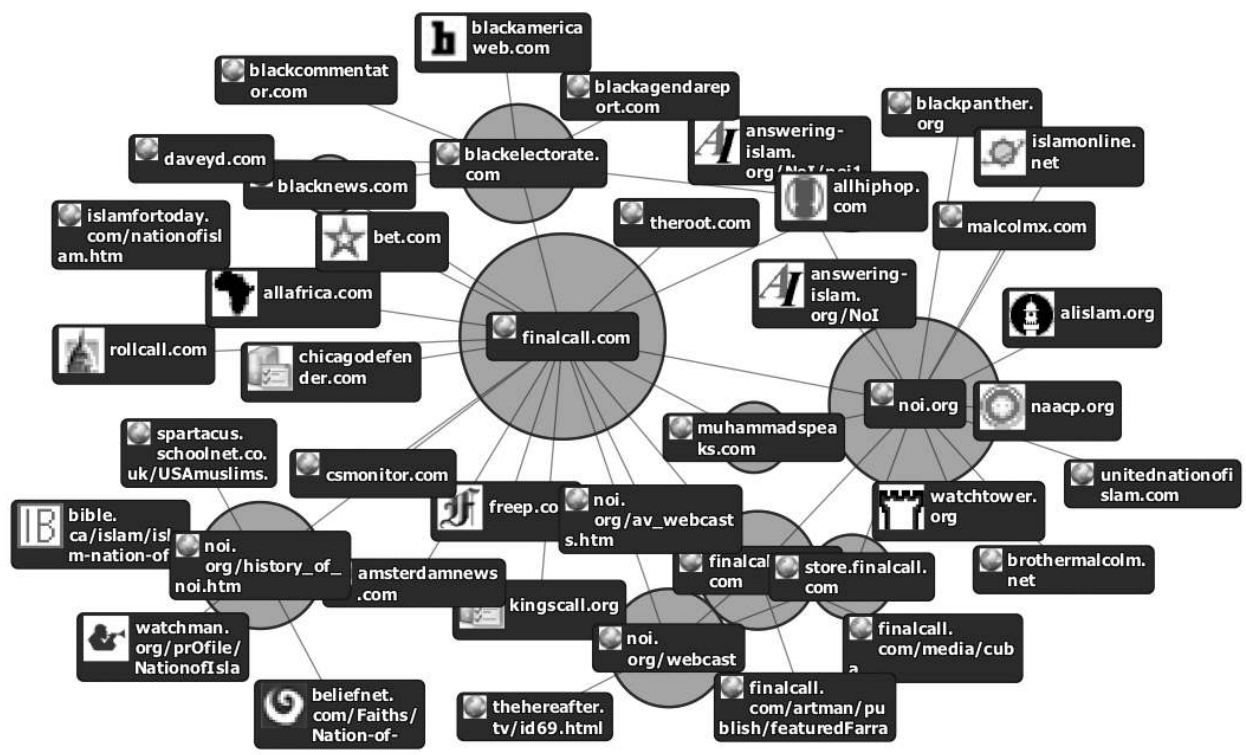

La France comble son retard avec les églises nouvelles de la Seine-SaintDenis. Elles trouvent un écho favorable auprès d'une certaine droite chrétienne française. Ces églises regroupées autour de la Fédération des églises pentecôtistes unies de France (FEPU) disposent de quelques sites Internet qui se développent rapidement ${ }^{13}$. La Nation de l'islam dispose en France de http://www.nationdelislam.com/ et en Grande-Bretagne de http://www.noi.org.uk/.

Le huitième groupe comprend des sites d'intellectuels, d'hommes de lettres, d'artistes et de sportifs. Ces sites ont la particularité de faire la promotion de personnalités des différentes communautés. Il s'agit en «mettant en avant» les individus de montrer des « modèles » à la jeunesse. Ils constituent une base biographique importante dans laquelle puisent éducateurs, parents, enseignants et jeunes. À travers ces portraits, il s'agit de renforcer l'image d'une communauté dynamique.

12 www.finalcall.com

13 www.epufrance.org/eglises.html 
La neuvième catégorie des sites Internet est celle des juristes. On retrouve des sites d'avocats, de conseillers juridiques, de conseillers matrimoniaux et des structures d'aide judiciaire. Ces sites sont des ressources pour faire face à un procès, à une incarcération ou à une réinsertion. L'incarcération des Noirs est considérée souvent comme un acte d'injustice sociale qui réveille la face sombre de l'histoire américaine. Les juges sont accusés de racisme et de discriminations. Les sites permettent de cerner la convocation et l'invocation du droit dans les rapports de domination qui traversent les États-Unis ${ }^{14}$.

Enfin, la dixième catégorie et sans doute la plus importante en nombre est celle des sites sur la beauté, la peau et le cheveu des femmes noires. Ces sites Internet sont une sorte de catalogue qui complète les magazines féminins dits ethniques soutenus par l'industrie du cosmétique. Le discours identitaire est ici fusionné aux symboles commerciaux. Le corps des femmes noires devient un enjeu commercial en raison du budget qu'elles consacrent aux soins de beauté. Le traitement des cheveux est, de loin, le plus important. Les industries cosmétiques exploitent largement la mode du défrisage lancée par des artistes noirs. Le passage des cheveux crépus coiffés à la « mode afro » des années 1970, aux cheveux défrisés des années 1980 souligne un changement d'époque. Il peut être interprété comme le passage d'une période des luttes pour les droits civiques à celle de la réussite par les affaires. Symboliquement, on passe de la coiffure «afro »d'Angela Davis à la coiffure défrisée d'Oprah Winfrey. Et les sites Internet ressemblent à des catalogues de vente où se succèdent des panneaux publicitaires (Byrds et Tharps, 1996).

Pour attirer la clientèle féminine on peut lire sur le site afrotherapy.com : «Afrotherapy est une boutique en ligne destinée à offrir une gamme exclusive des produits de beauté pour les cheveux et les soins de la peau noire. Notre objectif est d'offrir, de manière attentive, des produits de très haute qualité pour les cheveux et le soin de la peau noire. Et ce dans le monde entier pour satisfaire et résoudre les problèmes de cheveux de toutes les peaux noires et métissées à des prix abordables. (..) Nous comprenons votre défi de trouver le bon produit pour l'entretien de votre peau et de vos cheveux. Et c'est pour cette raison que nous ne stockons que des produits renouvelés afin de répondre à vos besoins individuels et spécifiques. Vous avez sans doute entendu parler de certaines marques et produits cosmétiques. Ils sont peut-être nouveaux pour vous, mais ont une seule chose en commun : ils ont été créés et testés pour répondre à vos besoins spécifiques ${ }^{15}$.

Le visage des femmes noires est photographié en gros plan : cheveux défrisés, bijoux, maquillage, couleur, sourire, beauté sont exploités par les publicitaires. Ces représentations contrastent avec les affiches publicitaires coloniales que décrit Blanchard (2001) : «Les visages en gros plan des personnages noirs, par exemple,

\footnotetext{
14 Pour exemple voir : http://www.hbdaniels.com, http://www.arkansasblacklawyers.com, http://www.blackexperts.com, http://www.blacklawyersforjustice.com, http://www.blackwomenlawyersla.org,

15 www.afrotherapy.com
} 
insistent sur les stéréotypes raciaux et accentuent l'altérité en la rendant presque monstrueuse. ${ }^{16}$ Mais les caractéristiques physiques caricaturées - les yeux en boule de loto, les lèvres lippues, les dents toutes dehors, le nez exagérément épaté -, qui sont associés à l'idée d'infériorité soulignée par le langage "petit nègre", signe de l'incapacité des Noirs à "assimiler" pleinement la culture française, sont absentes de la production officielle »(Blanchard, 2001).

À ces constructions se sont superposé d'autres plus récentes, empruntant à l'art, à la mode et à bien d'autres mouvements. Les femmes noires retournent le stigmate raciste en apportant des «corrections » et des retouches interprétées comme un progrès : le nez "désépaissi », la peau éclaircie et les cheveux défrisés. On ne trouve plus, comme dans les années 1960, des publicités pour les cheveux afro encore moins celles pour des tresses africaines. À la place, elles proposent des tresses industrielles et des perruques fabriquées en Chine et en Corée du Sud.

Sur le site de BlackHairmedia.com, on peut lire : « À Black Hair Media, nous voulons dédier ce site à toi et sur toi la femme noire, en t'apportant des idées que tu peux "essayer à la maison". Nous ne voulons pas consacrer notre site aux styles de coiffures ou encore aux salons de coiffure. Nous nous engageons à t'informer à partir de diverses publications, de livres et de notre propre recherche en ligne ».

Sur les publicités le corps des mannequins noirs est de plus en plus lisse et la peau «wetish» (couleur de blé) par opposition à la peau noire « rugueuse » des stéréotypes occidentaux. Le lissage du corps et le blanchissement de la peau s'inscrivent dans cette violence symbolique qui cherche l'harmonie avec le groupe dominant. Le laid disparaît en ce qu'il indispose, déstabilise et dérange. Les femmes à la recherche d'une certaine esthétique entrent ainsi dans le processus de marchandisation. Ces sites Internet participent, également, à la construction d'une identité. Les femmes cherchent à correspondre aux canons imaginés par l'industrie des cosmétiques et mis en œuvre par les publicistes. Le visage des femmes devient une sorte de tableau qui a pour fonction de représenter la «femme de son temps », la «femme noire occidentalisée ». L'Internet et le marchand jouent ici sur le détail. En revisitant les stéréotypes, ils leur donnent une double signification : légitimés par les «femmes noires » comme caractéristiques identitaires et acceptées par la société dominante. Sur les sites internet, les femmes sont fortement sollicitées : newsletters, boutiques en lignes, conseillers en cheveux, forum de discussion sur les cheveux, vendeurs de crèmes, «mentors en beauté » etc. En Grande-Bretagne et aux États-Unis, elles ont accès à «Black Hair Directory » qui consigne toutes les professions du cheveu : «Barbers, Beauty Shop, Beauty Product, Beauty School, Beauty Supply, Black Stylists, Blogs, Body Products, Books, Braids, Celebrity Hairstyles, Cosmetics, Cornrows, Curls, Curly Hair, Dreadlocks, Extensions, Forums, Haircare, Hair Blogs, Hair Books, Hair Forums, Hair Gallery, Hair Growth, Hair Loss, Hair Magazines, Hair Products, Hair Schools, Hair Shows, Kid's Haircare, Kid's Hairstyles, Kinky, Long Hairstyles,

16 Document $n^{\circ}$ 9/Affiche éditée par Gravier à l'occasion de l'Exposition Universelle de 1900, sur Le Transvallet l'Afrique sauvage. 
Magazines, Makeup, Medium Hairstyles, Men's Hair, Models, Nail Care, Natural Hairstyles, Oils \& Lotions, Salons, Short Hairstyles, Skincare, Twists, Weaves, Wigs ${ }^{17}$.

Le discours commercial s'appuie sur les thématiques du «bien-être », du «bien-vivre », de la «mode » et des «plaisirs» de la vie. Bien-être et bien-vivre se confondent et sont les prétextes aux affaires et aux plus-values. Cet ethno-capitalisme fait même disparaître le discours identitaire noir qui devient chromatique ou phénotypique, fondé sur la couleur de la peau et les caractéristiques physiques, centre d'intérêt de l'industrie cosméto-médiatique (Graphe 5).

Graphe 5 : Les sites Internet traitant de la beauté et des soins du corps des femmes noires

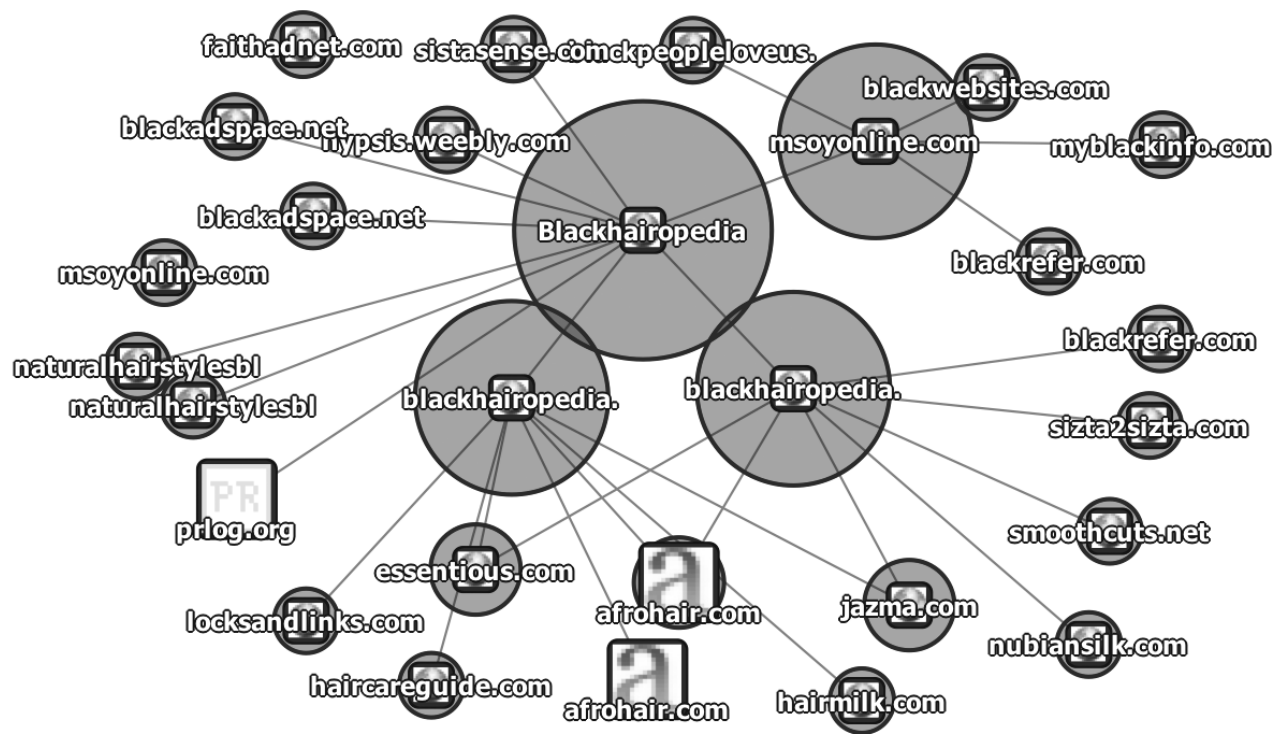

\section{UN SITE D'INFORMATION COMMUNAUTAIRE : WWW.GRIOO.COM}

Grioo.com est un site d'information communautaire, connu en France grâce à son forum de plus de 3000 membres créé en 2004. C'est aussi une entreprise dont la vocation est de produire de l'information sur les communautés noires de France et d'attirer les publicitaires. En quelques années, grioo.com s'est créé un véritable marché ethnique dont $80 \%$ des cybernautes sont en France. Il recueille : "Une présence internationale des internautes avec 77,9\% en Europe (dont $80 \%$ en France), 9,6\% en

17 www.myosonline.com. 
Afrique, 9,5\% en Amérique du Nord, 0,9\% en Asie, 0,2\% en Amérique du Sud et 0,1\% en Océanie. Une consultation très performante avec 287000 visiteurs uniques, 324000 visiteurs par mois, environ 1 million de pages vues par mois et une durée moyenne des visites supérieure à 9 minutes ${ }^{18}$. Il dispose aujourd'hui de quatre portails dont un consacré aux femmes « grioo pour elle ». Cependant, sa notoriété s'est faite grâce au forum des grionautes et dans lequel toutes les questions sur la communauté noire de France sont abordées. Son organisation et son mode de fonctionnement ressemblent à celui de www.Blackpanet.com, site Internet destiné aux Africains Américains et qui compte plus de 8 millions de membres, selon Nielsen Netrating ${ }^{19}$ une société de mesure et d'analyse des transactions sur Internet. Blackplanet est le site le plus visité par les Noirs américains comme grioo.com pourrait être le plus visible de la communauté noire de France. La logique des pseudonymes, dans les deux sites est comparable au cryptage en communication.

Cette Cybernégritude fonctionne par « ratissage » (Beck, 2004) et « extraversion culturelle ». Elle invite dans ses forums, un public noir éclectique et élargi : cadres, ouvriers, intellectuels, entrepreneurs... Ses agents médiatiques usent de toutes les ressources et outils disponibles sur Internet. Le lien hypertexte leur permet, ainsi, de voyager et de récupérer une quantité importante de données sur les Noirs dans le monde. Ils n'hésitent pas à copier la mise en page, les articles et les discours pour construire un « point de vue noir ».

C'est de manière affective et émotionnelle que les forumistes choisissent leurs pseudonymes. L'écrasante majorité puise au registre des noms propres, des noms de lieux et des formules atypiques. Le forum est un lieu de discussion des sujets internes et externes aux communautés noires. Il est surtout le lieu d'une expression des «identités collectives anonymes ». Le pseudonyme est à la fois un moyen de se cacher aux siens mais aussi à la société dominante. En s'y inscrivant pour discuter des conditions des populations noires de France et du monde, le forumiste porte un message, qu'il expose sur cette place publique sans se mettre en danger. Les forumistes traitent de tous les sujets notamment ceux qui dominent l'actualité française. De l'affaire Dieudonné aux émeutes des banlieues, les forumistes ont tenu un journal de bord des populations noires en France. En quatre ans, les 20 premiers forumistes de grioo.com ont écrit plus de 20000 messages dont plus de 1000 chacun (voir tableau 1). C'est donc un véritable activisme communautaire où s'exercent une stratégie et une tactique qui commencent par le choix du pseudonyme. Derrière chaque pseudonyme, on retrouve à la fois les enjeux de l'auto-désignation mais aussi les paradigmes de représentation (voir Graphe 6).

Le premier groupe de pseudonymes est constitué de prénoms africains comme «Adebisi », «Abiola », «Ado-aro », «Agnassi », etc. Les prénoms du Golfe du Bénin sont privilégiés. Certains pseudonymes reprennent des lieux et des nationalités comme « Douala », « Bamiléké », « Yoruba », « Bantu Kelani », « Bahia », « Kilombo »,

18 www.grioo.com

19 http://en-us.nielsen.com/home. 
Tableau 1 : Les dix premiers forumistes de www.grioo.com entre 2004 et 2009

\section{grioo.com \\ Espace de discussion}

(3)

(8) Profil @Se connecter pour vérifier ses messages privés @ Connexion

\begin{tabular}{|c|c|c|c|c|c|c|c|}
\hline $\begin{array}{l}\text { grio } \\
\text { Ind } \\
\text { For }\end{array}$ & S du Séle & ctionner la méthode & de tri: & Top 10 des Posteurs & Ordre & Décroissant & \begin{tabular}{|l} 
Trier \\
\end{tabular} \\
\hline$\#$ & & Nom d'utilisateur & E-mail & Localisation & Inscrit le & Messages & Site Web \\
\hline 1 & $80 \mathrm{mp}$ & Chabine & & & $\begin{array}{l}02 \mathrm{Mar} \\
2005\end{array}$ & 3805 & \\
\hline 2 & $80 \mathrm{mp}$ & Maryjane & & Derrière toi & $\begin{array}{c}25 \mathrm{Mai} \\
2005\end{array}$ & 3325 & (6) www \\
\hline 3 & $80 \mathrm{mp}$ & M.O.P. & & & $\begin{array}{c}11 \mathrm{Mar} \\
2004\end{array}$ & 2312 & \\
\hline 4 & $80 \mathrm{mp}$ & ARDIN & & UK & $\begin{array}{l}22 \text { Fév } \\
2004\end{array}$ & 1998 & (6) www \\
\hline 5 & $88 \mathrm{mp}$ & Muana Kongo & & & $\begin{array}{l}09 \mathrm{Mar} \\
2004\end{array}$ & 1867 & (6) www \\
\hline 6 & $80 \mathrm{mp}$ & Pakira & & & $\begin{array}{l}01 \mathrm{Mar} \\
2004\end{array}$ & 1866 & \\
\hline 7 & $88 \mathrm{mp}$ & francois & & IDF & $\begin{array}{l}14 \text { Déc } \\
2004\end{array}$ & 1810 & (6) www \\
\hline 8 & $80 \mathrm{mp}$ & Soundjata Kéita & & $\begin{array}{c}\text { Au sein de mon } \\
\text { Empire }\end{array}$ & $\begin{array}{l}06 \mathrm{Mai} \\
2005\end{array}$ & 1723 & \\
\hline 9 & $82 \mathrm{mp}$ & Kainfri & & & $\begin{array}{l}22 \text { Fév } \\
2004\end{array}$ & 1693 & \\
\hline 10 & $80 \mathrm{mp}$ & zingh 2006 & & & $\begin{array}{l}04 \text { Jan } \\
2006\end{array}$ & 1533 & \\
\hline
\end{tabular}

Graphe 6 : www.grioo.com et ses ressources dans la fabrication de l'information

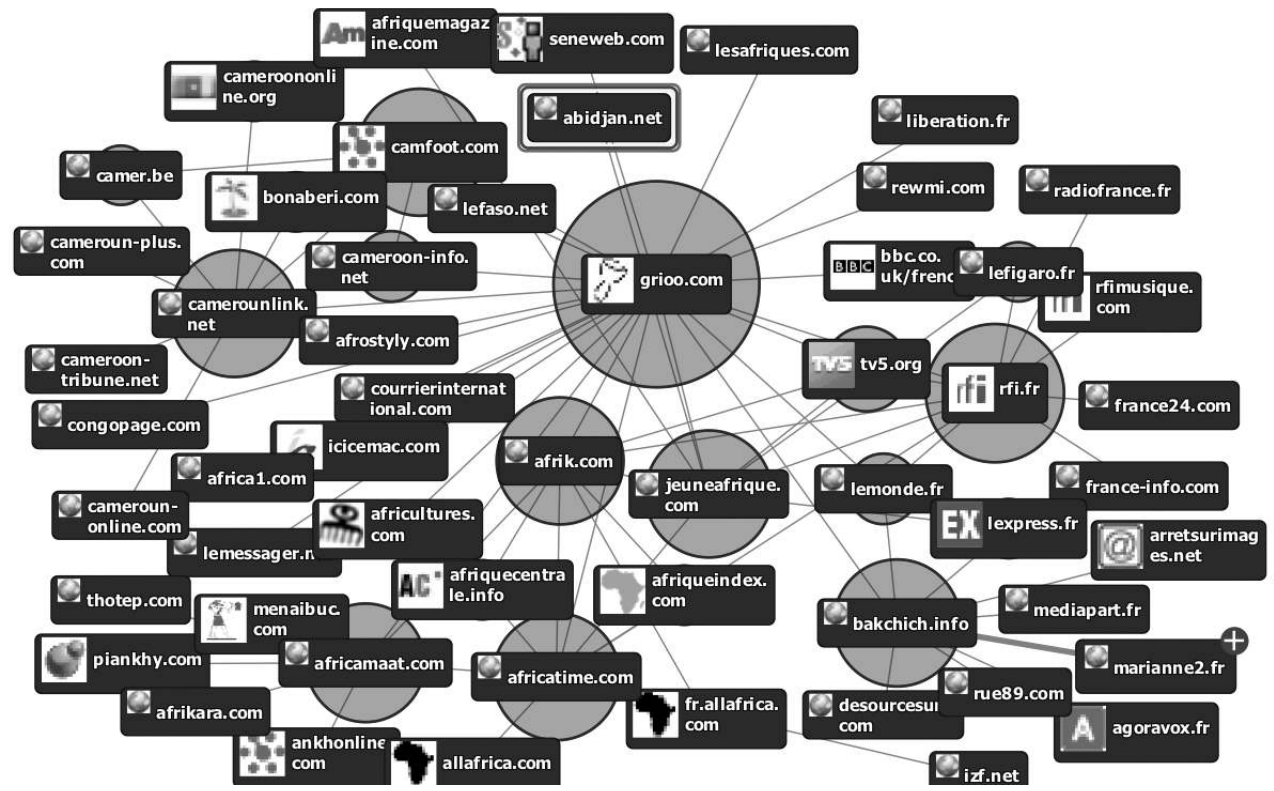


«Owambo », « Africain », « Africaine », « African libre », « Afrobeat », « Afrocalipse », « fille d'Afrique », « Gondowana », «Panafricain » etc. Le troisième regroupe des personnages historiques dont le dénominateur commun est le courage, le nationalisme, la virtuosité : «Hannibal », «Shaka Zulu », «Kunta Kinte ${ }^{20}$ », «Soundjata Kéita », «Queen Pokou », «Louverture-Césaire », « Malcolm », « Steve Biko », « Chevalier de Saint-Georges » et «Ogotommeli » (grand maître de la parole dogon au Mali) à qui Marcel Griaule dans Dieu d'eau (Fayard, 1975) rend hommage. Outre ces pseudonymes prestigieux des centaines d'autres font référence à l'Afrique et à ses diasporas. Nous trouvons aussi des références à l'Égypte pharaonique et antique dans les pseudonymes de «Ankh» la croix symbole de la vie, les noms de « Nefertity » la princesse, «Shabaka » le roi, «Imhotep » le savant, "Akenaton » le pharaon et «Kemet » le nom originel du pays des pharaons. L'Institut africamaat et les Éditions Menaibuc ont publié nombre d'ouvrages sur l'Egypte, source d'inspiration pour les forumistes. En puisant dans l'histoire et la géographie, les internautes noirs se dissimulent à la société dominante et ils « déstabilisent » en cryptant leur mode d'apparition dans l'espace public virtuel. Ils brouillent les pistes en « africanisant » leurs pseudonymes et compliquent la tâche des détracteurs, des traceurs éventuels et des polices électroniques. Le pseudonyme est le costume du cyberactiviste. Il se présente comme une stratégie de résistance à la société dominante, ses codes et ses règles. Le forum devient l'agora et une scène de contestation. Dans les noms des sites, les références aux territoires les plus utilisés sont liées à l'Afrique. Le mot «Afrique » se trouve dans 141 sites sous diverses déclinaisons (Afri, Africa, Afrik, Afrique, Afro, Angola, Burundi, Cameroun, Congo, etc.). Symboliquement, le signifiant « Afrique » est utilisé pour signifier l'attachement aux racines.

Le second terme dominant dans la dénomination des sites Internet est celui de «Black» associé quelques fois à Beauty, Hair, Hiphop, Reggae, Sister, Urban. Le mot «Black » est utilisé par 119 sites et suivi de « urban » par 11 sites Internet, Ebony sur 8 sites. Le terme «Black» a un sens familier qui désigne cet autre qui me ressemble c'est-à-dire le cybernaute noir. La symbolique phénotypique a aussi une dimension publicitaire et vise autant l'internaute ordinaire que les élites. De nombreux sites commerciaux utilisent une dénomination phénotypique pour faire la publicité de leurs affaires. «Black » a d'une certaine façon à la fois une connotation identitaire et commerciale.

Le terme « urban » revient aussi fréquemment dans les sites pour s'adresser aux jeunes et dans une certaine mesure aux amateurs de musiques dites urbaines. La référence à l'espace urbain tend aussi à désigner les zones défavorisées où vivent les populations noires et immigrées en général. Le mouvement Hiphop est porteur de ces sites qui célèbrent la création artistique de la jeunesse urbaine. Dans les trois cas décrits, on relèvera que les concepteurs des sites transforment un "stigmate » en marqueur identitaire pour toucher l'internaute. Le procédé est identique lorsque l'Internet féminin retravaille les stéréotypes et stigmates pour les charger de symboliques identitaires.

20 Kunta Kinte, personnage historique de Racines (CEDA, 1981) d'Alex Haley raconte l'épopée d'un guerrier mandingue devenu esclave. C'est une figure emblèmatique des nègres marrons. 


\section{CONCLUSION}

La cartographie des références identitaires peut être envisagée comme une traduction d'usages mis en place par les communautés noires. Ces usages ont une dimension «structurante » et sont construits par les cybernautes. L'Internet a pénétré la vie des gens qui l'ont forgé et utilisé. Tout se passe comme si l'Internet était le lieu privilégié des expressions identitaires et politiques. Qu'il s'agisse du « web diasporique directif », « participatif » ou « personnel », les usages restent complémentaires. La facilité et la malléabilité de l'espace virtuel permettent de passer de l'un à l'autre. Le cyberespace permet une circulation sans contrôle des opinions, des discours en se jouant des frontières. L'Internet des diasporas noires est dans un sens le reflet de leurs conditions socio-économiques et de leur environnement. L'Internet a « transnationalisé » et déterritorialisé les offres commerciales. De même qu'il a « transnationalisé » et déterritorialisé la théologie de la prospérité, «supplément » idéologique du néolibéralisme même si ce processus est plus vrai dans le monde anglophone que dans le monde francophone : la cybernégritude reste très influencée par le modèle américain. Le cybermonde noir se définit comme un véritable « maquis des diasporas noires », en quête d'émancipation et de reconnaissance. Il convoque et mélange les paradigmes, les revendications et les références à Martin Luther King, à Malcolm X, à Jesse Jackson, à Asante Molefi à Louis Farakhan, à Oprah Winfrey, à Michael Jackson et enfin à Michelle ou Barack Obama. Il évoque et convoque toutes les périodes historiques. Le Black style de l'Internet noir de Paris et Londres ne serait-il pas, un « prêt-à-penser » un «prêt-à-croire » dérivé de l'american way of life, et donc, dans une certaine mesure un produit de la société de consommation dans un emballage africanisé ? Il semble que l'« ethnoscape » (Appadurai, 1997) à l'intérieur duquel évolue les diasporas noires ne s'éloigne guère d'une logique ethnocapitaliste.

L'analyse des articles et des rubriques ainsi que de l'organisation des forums et de la mise en scène de l'information, met en évidence, ce que l'on pourrait appelé « un cosmopolitisme obligé ». L'Internet des diasporas noires réinvente en quelque sorte une « utopie noire mondialisée ». Comme aux États-Unis, on observe en France et en Grande-Bretagne un même souci de réinterpréter ou de réinventer ce qui est considéré comme des traditions africaines, de faire appel aux sentiments et ressentiments pour construire une culture transnationale pour soi et exposée aux regards de l'autre.

Avec l'Internet et ses innombrables applications et solutions de communications, la possibilité d'être à la fois dans la « globalité » et la « localité » permet de plus en plus de « circuler » dans un monde que n'arrêtent pas les frontières. 


\section{Références bibliographiques}

ALKALIMAT Abdul (2004) The African American Experience in Cyberspace, London, Viginia, Pluto, 293 p.

ALKALIMAT Abdul (2007) Social cyberpower in the everyday life of african american community : a report on action-research in Toledo, Ohio, Urban Affairs Center, 31 p.

APPADURAI Arjun (1997) Après le colonialisme, Payot, 334 p.

APPADURAI Arjun (1990) Disjuncture and difference in the Global cultural economy, in M. Featherstone (ed), Global Culture. Nationalism, Globalization and Modernity, London, Sage, pp. 295-310.

ASSELIN Christophe (2007) E-Réputation, quand la toile ne se contente plus d'écouter, Journal du Net. Disponible sur http://www.journaldunet.com.

BECK Ulrich (2003) Pouvoir et contrepouvoir à l'heure de la mondialisation, Paris, Aubier, $600 \mathrm{p}$.

BERNAL Victoria (2006) Diaspora, cyberspace and political imagination: the Eritrean diaspora online, The Authors, pp. 161-179.

BERTHOMIERE William et CHIVALLON Christine (éds.) (2006) Les diasporas dans le monde contemporain : un état des lieux, Paris, Karthala, 419 p.

BHABHA Homi K. (2007) Les lieux de la culture, Paris, Editions Payot \& Rivages, 411 p.

BLANCHARD Pascal (2001) La représentation de l'indigène dans les affiches de propagande coloniale entre concept républicain, fiction phobique et discours racialisant, Hermès, 30, pp. 149-168.

BOLTANSKI Luc, CHIAPIELLO Eve (1999) Le nouvel esprit du capitalisme, Paris, Gallimard, $843 \mathrm{p}$.

BOURDIEU Pierre (2001), Langage et pouvoir symbolique, Paris, Seuil, 423 p.

BYRD D. Ayana, THARPS L. Lori (2001) Hair Story : untangling the roots of black hair in America, New York, St. Martin Griffin, 198 p.

BYRNE D. N. (2007) Public discourse, community concerns, and civic engagement: Exploring black social networking traditions on BlackPlanet.com, Journal of Computer-Mediated Communication, 13(1), article 16. http://jcmc.indiana.edu/vol13/issue1/byrne.html.

CERTEAU (de) Michel (1990) L'invention du quotidien, tome 1 : arts de faire, Paris, Gallimard, $350 \mathrm{p}$.

COHEN Robin (2006) "Diaspora : changing meanings and limits of the concept", in BERTHOMIERE William et CHIVALLON Christine (éds.) (2006) Les diasporas dans le monde contemporain : un état des lieux, Paris, Karthala, pp. 40-48.

FOURCHARD Laurent, MARY André, OTAYEK Réné (2005) Les Entreprises religieuses transnationales en Afrique de l' Ouest, Paris, Karthala, 537 p.

FATH Sébastien (2008) Dieu XXL. La révolution des mégachurches, Paris, Autrement, 192 p.

FRIEDMAN Thomas (2006) La terre est plate, Paris, Saint Simon, 283 p.

Gilroy Paul (2003) L'atlantique noir. Modernité et double conscience, Paris, Kargo, 333 p.

GUMBS Bob (1999) Internet directory to black web sites, Cultural expression Inc. New York. $103 \mathrm{p}$.

GOFFMAN Erving (1973) La mise en scène de la vie quotidienne. Tome 1, La présentation de soi, Paris, Les éditions de minuit, $256 \mathrm{p}$.

HAENNI Patrick (2005) L'islam de marché, Paris, Seuil, 140 p.

HALL Stuart (1997) Representation: Cultural Representations and Signifying Practices, London, Sage 391 p.

HALL Stuart (1993) Cultural identity and diaspora, in In Williams, Patrick \& Laura Chrisman eds. Colonial Discourse \& Postcolonial Theory: A Reader. Harvester Whaeatsheaf, pp. 223237. 
HELLAND C. (2007) Diaspora on the electronic frontier: Developping virtual connections with sacred homelands, Journal of Computer-Mediated Communication, 12(3), article 10. http://jcmc.indiana.edu/vol12/issue3/helland.html.

KARIM H. Karim (2003) The Media of Diaspora, London, New York, Routledge, 236 p.

LAHIRE Bernard (2005) L'esprit sociologique. Paris, La Découverte, 434 p.

LATOUR Bruno, WOOLGAR Steve (1990) La vie de laboratoire, Éditions la Découverte, Paris $308 \mathrm{p}$.

MANDAVILLE Peter G. (2001) Transnational Muslim Politics: Reimagining the Umma, George Mason University Press, 256 p.

MARY André, CORTENS André (éds.) (2000) Imaginaires politiques et pentecôtismes, Paris, Karthala, 368 p.

MATTELART Armand (2002) La mondialisation de la communication, Paris, PUF, 123 p.

MODOOD Tariq (2009), Multicultural Politics: Racism, Ethnicity and Muslims in Britain. University of Minnesota Press and Edinburgh University Press, $240 \mathrm{p}$.

NADJE Al-Ali, KHALED Koser (2001) New Aproaches to Migration? Transnational Communities and the Transformation of Home, London \& New York, Routledge, $226 \mathrm{p}$.

REX, John (1986), Race and ethnicity. Milton Keynes, Open University Press, 148 p.

SAID Edward (1994) Culture and Imperialism, London, Vintage $444 \mathrm{p}$.

SINGLY (de) François (2005) L'individualisme est un humanisme, La Tour d'Aigues, Éditions de l'Aube, $127 \mathrm{p}$.

VERHULST Stefaan (1999) Diasporic and transnational communication: technologies, policies and regulation, The Public, Vol 6, 1. 29-36.

WEBSTER Frank (ed.) (2001) Culture and Politics in the Information Age, Birmingham, University of Birmingham Press, $256 \mathrm{p}$.

ZALESKI J. (1997) The Soul of Cyberspace: How New Technology is Changing our Spiritual Lives, New York, Harper Collins, 284 p. 